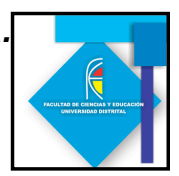

\title{
SECUENCIA DE ENSEÑANZA PARA LA PRESENTACIÓN DEL ANÁLISIS DEL FENÓMENO DE INDUCCIÓN ELECTROMAGNÉTICA
}

\section{SEQUENCE OF INSTRUCTION FOR THE PRESENTATION OF ANALYSIS OF THE PHENOMENON OF ELECTROMAGNETICINDUCTION}

\author{
Oyuela Mateus Diana Isabel ${ }^{1}$ \\ Isabel Garzón Barragán²
}

\section{Resumen}

En este trabajo se desarrolla una secuencia de enseñanza basada en la metodología de enseñanza-aprendizaje por investigación orientada, para presentar el análisis del fenómeno de inducción electromagnética en un curso introductorio de electromagnetismo para estudiantes de nivel universitario. Esta secuencia se fundamenta en dos premisas; una es que "todo conocimiento es la respuesta a una pregunta" y la otra es que los estudiantes construyen su conocimiento desde su propia experiencia, la cual es generada por los ambientes de aprendizaje que los profesores propician.

Con el fin de que los estudiantes alcancen un aprendizaje significativo de las explicaciones macroscópica y microscópica del fenómeno de inducción electromagnética, en la secuencia de enseñanza se consideran aspectos como, la relación que guarda la fem inducida con la variación del flujo magnético en el tiempo a través de un circuito, la descripción del fenómeno en términos de fuerzas y el vínculo que existe entre la fem inducida y las medidas de la corriente y el voltaje en un circuito.

Cabe resaltar que este artículo hace parte de un trabajo de grado que se está desarrollando, por esta razón la implementación de la secuencia es una tarea que se realizará en el futuro.

Palabras clave: Secuencia de enseñanza, inducción electromagnética y fem inducida.
Abstract
In this paper develops a teaching sequence based on the teaching-learning approach by oriented research, to present the analysis of the phenomenon of electromagnetic induction in an introductory course on electromagnetism. This sequence is based on two premises:

\footnotetext{
1 Departamento de física, Universidad Pedagógica Nacional, Bogotá D.C, Colombiadfi33_doyuela@pedagogica.edu.co

2 Departamento de física, Universidad Pedagógica Nacional, Bogotá D.C, Colombia, igarzon@pedagogica.edu.com
} 


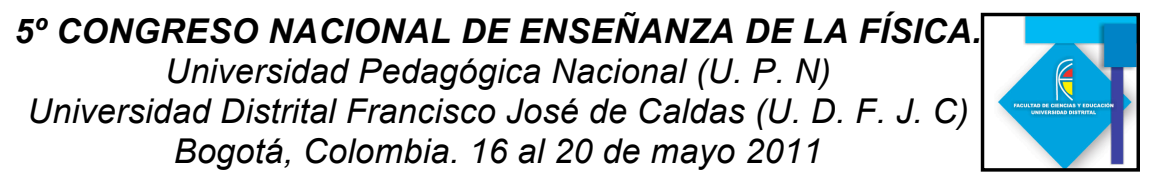

one is that "all knowledge is the answer to a question" and the other is that students construct their knowledge from their own experience, which is generated by the learning environments that foster teacher.

In order that students achieve significant learning and macro explanations of the phenomenon of electromagnetic induction in the sequence are considered aspects such as education, the relationship of the emf induced magnetic flux variation in time through a circuit, the description of the phenomenon in terms of forces and the bond that exists between the induced emf and the measurements of current and voltage in a circuit.

It should be noted that this article is part of a thesis being developed, for this implementation of the sequence is a task that will take place in the future.

Keywords: Teaching sequence, electromagnetic induction, induced emf.

\section{Introducción}

La mayoría de los estudiantes considera el electromagnetismo como un tema difícil y poco atractivo. La investigación ha mostrado de manera reiterada, el escaso aprendizaje de los estudiantes después de la enseñanza en dicho campo (Guisasola 2008). En este artículo se propone una secuencia de enseñanza para presentar la explicación del fenómeno de inducción electromagnética, basada en la metodología enseñanza-aprendizaje por investigación orientada, en la secuencia se plantean situaciones problemáticas que buscan conquistar el interés de los estudiantes y la participación de forma activa en la construcción de su conocimiento.

Los aspectos de la metodología que incluye esta secuencia son los siguientes: el estudio cualitativo del fenómeno de inducción electromagnética, la emisión de hipótesis por parte de los estudiantes especialmente en la explicación de los montajes experimentales, la elaboración de estrategias de resolución como experimentos y finalmente la comunicación y discusión del tema en el aula de clase, concibiendo la interacción social como una forma de aprender.

La secuencia de enseñanza está constituida por una serie de preguntas, actividades y situaciones diseñadas con el fin de buscar solución a un conjunto de dificultades que los alumnos universitarios muestran a la hora de analizar el fenómeno de inducción electromagnética. Gran cantidad de investigaciones responsabilizan de estas dificultades a las deficiencias de los estudiantes, en este documento se tiene en cuenta que uno de los factores que más influye en el aprendizaje es el cómo se presenta la explicación del fenómeno. En el caso de la fuerza electromotriz inducida, esta se suele presentar como si se tratará de dos clases de fem (fem de movimiento y fem de transformación) totalmente individuales y adicionalmente se ignoran algunas explicaciones, como las tres causas de la variación del flujo magnético en el tiempo, que hacen parte de lo que se quiere mostrar en este trabajo. 


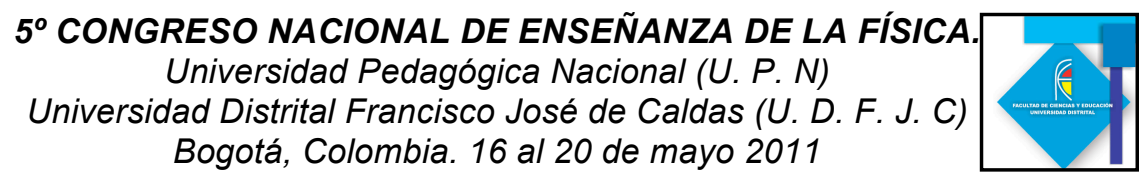

\section{Dificultades en el aprendizaje a superar con el diseño de la secuencia.}

Recientes investigaciones muestran dificultades de los estudiantes universitarios en el aprendizaje de la explicación del fenómeno de inducción electromagnética (Galili, 2006; Guisasola, 2010; Thong, 2008). Con la secuencia de enseñanza se pretende superar algunas de esas dificultades:

1. La confusión entre la presencia de líneas de campo que atraviesan el circuito y la variación del flujo magnético en el tiempo a través del circuito.

2. La omisión de una explicación en términos de fuerzas (explicación microscópica) de los fenómenos de inducción electromagnética.

3. La confusión entre el área del circuito y el área de integración en la ley de Faraday, cuando se analiza experimentos de inducción electromagnética de movimiento

La tercera dificultad se refiere a la forma incorrecta de razonar sobre la variación de la superficie del flujo magnético, esta superficie se debe entender como el área barrida por el circuito móvil en un intervalo de tiempo y no confundirse con el área del circuito, de lo contrario se cae en el error de considerar la ausencia de una corriente inducida cuando no existe variación de campo magnético o variación del área del circuito.

\section{Estructura de la secuencia de enseñanza}

A continuación se presentan las preguntas, actividades y situaciones que componen la secuencia de enseñanza y el objetivo especifico de cada una de ellas.

\begin{tabular}{|c|c|}
\hline PREGUNTA O ACTIVIDAD & OBJETIVO \\
\hline $\begin{array}{l}\text { ¿Cómo hacer que circule } \\
\text { corriente por un circuito sin } \\
\text { hacer uso de una batería? }\end{array}$ & $\begin{array}{l}\text { Con esta pregunta además de inquietar a los alumnos, se } \\
\text { pretende conocer las nociones básicas que ellos poseen sobre } \\
\text { inducción electromagnética, en especial la relación entre la } \\
\text { electricidad y el magnetismo, se piensa en esta relación porque } \\
\text { fue uno de los problemas que se hizo presente en la historia de } \\
\text { la ciencia. }\end{array}$ \\
\hline $\begin{array}{l}\text { Representar en un } \\
\text { esquema el diseño de un } \\
\text { circuito por el que circule } \\
\text { corriente eléctrica sin hacer } \\
\text { uso de una batería. }\end{array}$ & $\begin{array}{l}\text { Se pretende que los estudiantes propongan hipótesis que } \\
\text { posteriormente puedan corroborar o anular en las experiencias } \\
\text { planteadas por el profesor. En esta actividad se espera que los } \\
\text { estudiantes hablen del funcionamiento que tiene cada } \\
\text { instrumento que ponen en el circuito. }\end{array}$ \\
\hline
\end{tabular}

A Situación 1: Un imán atraviesa una espira de alambre conductor con una velocidad , el galvanómetro marca el valor de la corriente inducida que se genera en la espira. 

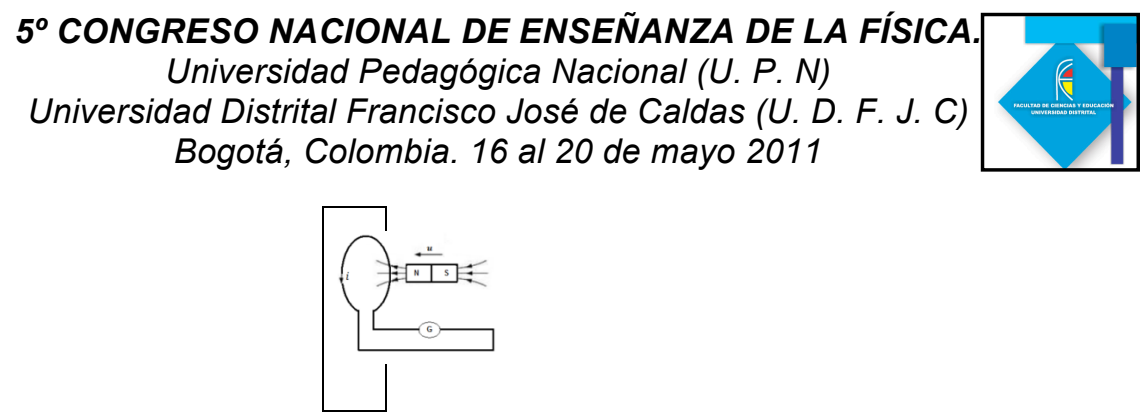

Figura1. Situación 1

\begin{tabular}{|c|c|}
\hline PREGUNTA $O$ ACTIVIDAD & OBJETIVO \\
\hline $\begin{array}{l}\text { ¿Qué magnitudes físicas } \\
\text { actúan como variables en el } \\
\text { circuito? y ¿Cuáles como } \\
\text { constantes? }\end{array}$ & $\begin{array}{l}\text { Bajo la observación del experimento A planteado por el profesor } \\
\text { se espera que los estudiantes piensen en la caracterización del } \\
\text { circuito utilizando conceptos previos, especialmente el de flujo } \\
\text { magnético. Con esto se obtiene una primera aproximación a la } \\
\text { ley de inducción de Faraday. }\end{array}$ \\
\hline $\begin{array}{l}\quad \text { ¿Cómo se relaciona la fem } \\
\text { inducida con el flujo } \\
\text { magnético para describir lo } \\
\text { observado? }\end{array}$ & $\begin{array}{l}\text { Esta pregunta se hace después del análisis macroscópico, con el } \\
\text { fin de que los estudiantes establezcan la relación entre las } \\
\text { variables que para ellos caracteriza el circuito. }\end{array}$ \\
\hline $\begin{array}{l}\text { ¿Cuál es la causa del } \\
\text { movimiento de los electrones } \\
\text { en la corriente inducida? } \\
\text { Explique su respuesta en } \\
\text { términos de fuerzas. }\end{array}$ & $\begin{array}{l}\text { Con este tipo de preguntas se procura mostrar un análisis } \\
\text { microscópico de los fenómenos de inducción, con el fin de que } \\
\text { los alumnos complementen la explicación macroscópica que está } \\
\text { dada en términos del cambio de flujo magnético. }\end{array}$ \\
\hline 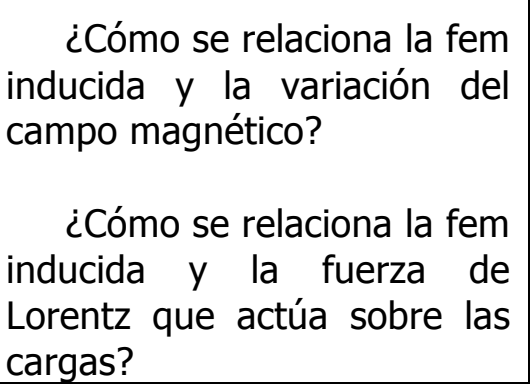 & $\begin{array}{l}\text { Con estas dos preguntas se pretende que los estudiantes } \\
\text { establezcan relaciones entre las variables que caracterizan el } \\
\text { circuito. Además es una forma de indagar las nociones que } \\
\text { tienen los estudiantes, en este caso sobre campos eléctricos, } \\
\text { campos magnéticos sus variaciones y la fuerza de Lorentz. }\end{array}$ \\
\hline
\end{tabular}

En esta situación también se puede mover la espira y dejar el imán en reposo, de esta manera los estudiantes tiene la oportunidad de visualizar que se utilizan dos formas diferentes para general la fem, aunque el valor numérico de esta sea invariante

Debido a que el cambio de flujo magnético en el tiempo se genera de tres maneras diferentes, se presenta un conjunto de situaciones que le permita al estudiante identificar y comprender las razones por las que se genera variación del flujo magnético en el tiempo: 
$5^{\circ}$ CONGRESO NACIONAL DE ENSEÑANZA DE LA FÍSICA

Universidad Pedagógica Nacional (U. P. N)

Universidad Distrital Francisco José de Caldas (U. D. F. J. C)

Bogotá, Colombia. 16 al 20 de mayo 2011
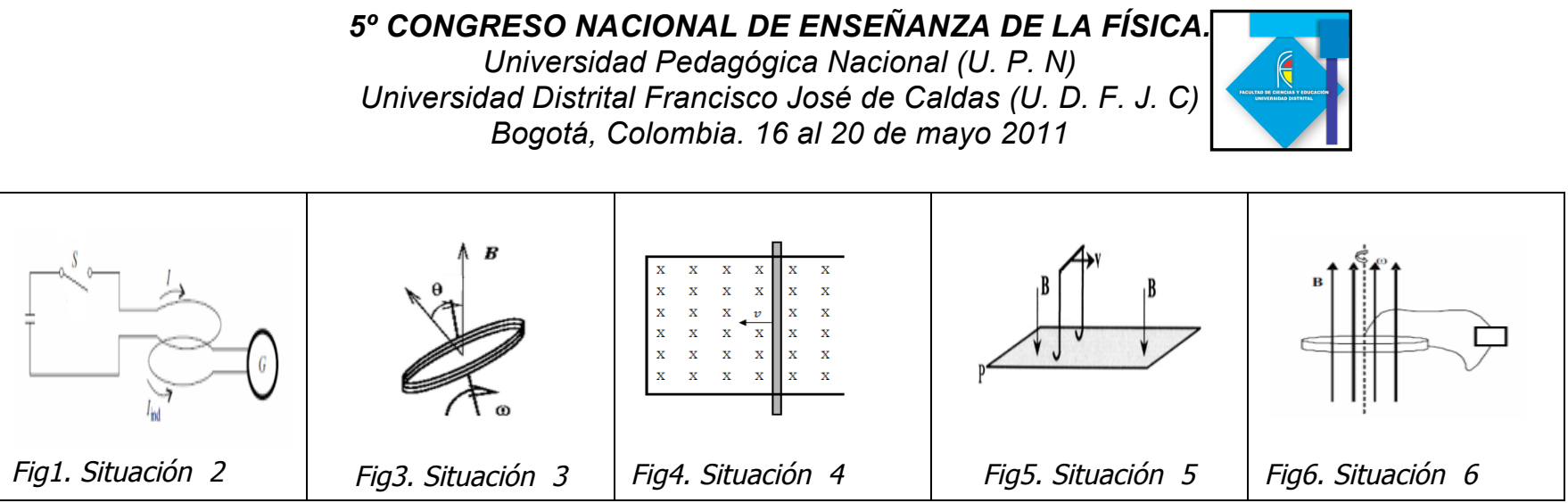

B En este apartado se considera el caso en el que la variación del campo magnético produce la variación del flujo magnético en el tiempo.

Situación 2: por la espira superior pasa una corriente porque está conectada a una fuente, cada vez que se cierra y se abre el circuito la aguja del galvanómetro sufre una desviación.

C Con los siguientes experimentos se pretende estudiar el caso en el que la variación del área del circuito produce la variación del flujo magnético en el tiempo. Adicionalmente se puede hacer un análisis microscópico utilizando la fuerza de Lorentz que actúa sobre el conjunto de cargas que componen el alambre conductor del circuito.

Situación 3: Una bobina de pocas espiras gira con una velocidad angular en el interior de un campo magnético uniforme, un galvanómetro puesto en sus extremos indica el paso de la corriente inducida.

Situación 4: En un campo magnético uniforme se pone un alambre de conducción en forma de $U$ (Fig4) y un travesaño que se mueve hacia la izquierda, la variación de la posición del travesaño genera una fem inducida en el circuito.

D Con estos fenomenos se intenta eliminar la dificultad de confundir el area del flujo magnetico que aparece en la ley de Faraday con el area del circuito. La intension es que los estudiantes argumenten la variación del flujo magnético en el tiempo con la variación del área de integración en la ley de Faraday.

Situación 5: un alambre en forma de $U$ viaja verticalmente sobre una plataforma horizontal, en medio de un campo vectorial (Fig5).

Situación 6: GENERADOR UNIPOLAR DE FARADAY. Un disco gira con velocidad angular a través de un imán, se conecta un galvanómetro que detecta la corriente inducida con uno de sus extremos en el eje del disco y el otro en uno de los extremos.

\begin{tabular}{|c|c|}
\hline \multicolumn{2}{|c|}{ La siguiente pregunta se hace después de presentar las situaciones $2,3,4,5$ y 6 . } \\
\hline PREGUNTA O ACTIVIDAD & OBJETIVO \\
\hline $\begin{array}{l}\text { ¿Qué cambios en las } \\
\text { opiedades o características }\end{array}$ & $\begin{array}{l}\text { Con esta pregunta se desea que los } \\
\text { tres razones diferentes para produ } \\
\text { magnético en el tiempo. }\end{array}$ \\
\hline
\end{tabular}
electromotriz inducida?

\section{Consideraciones finales}

Cabe resaltar que la implementación de esta ponencia aun no ha sido realizada y se proyecta como una tarea para el futuro, por lo tanto la implementación de la propuesta queda abierta para los profesores que anhelan enseñar la explicación del fenómeno de 


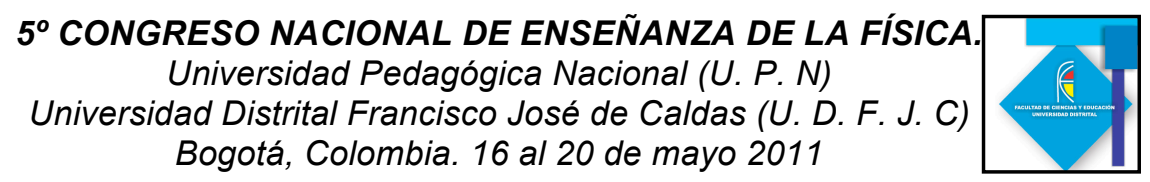

inducción electromagnética. La recomendación para estos profesores es que en las orientaciones que ofrece a sus estudiantes propicie ambientes que generen interés en ellos.

La secuencia promueve un cambio en la presentación que se está abordando de la inducción electromagnética en los cursos de inducción al electromagnetismo, la decisión está en el docente de emplear completamente la secuencia o modificarla según las necesidades y problemáticas que descubra en sus estudiantes, como la persistencia en conceptos previos y el uso no adecuado de los instrumentos de laboratorio.

Para el uso de esta secuencia es necesario conocer las dificultades que se pretenden superar de la enseñanza tradicional sobre la inducción electromagnética y las diferentes causas que se plantean como justificación de la variación del cambio del flujo magnético, además de revisar los objetivos específicos que contiene cada componente de la ponencia.

\section{Referencias Bibliográficas}

[1] GALILI, I. KAPLAN, D. LEHAVI, Y. (2006). Teaching Faraday's law of electromagnetic induction in an introductory physics course. American Journal of Physics, 74(4), pp 337343.

[2] GUISASOLA, J.; ALMUDI, J.; MANUEL, J. y MIKEL, C. (2008). Dificultades persistentes en el aprendizaje de la electricidad: estrategias de razonamiento de los estudiantes al explicar fenómenos de carga eléctrica. Revista Brasileira de Ensino de Fisica, 32(1), pp 1401.

[3] GUISASOLA, J; ALMUDI, J. y ZUZA, K. (2009). Dificultades de los estudiantes universitarios en el aprendizaje de la inducción electromagnética. Revista Brasileira de Ensino de Fisica, 32(1), pp 1401.

[4] MARAZZINI, P; TUCCI, P. (1983). The physical meaning of Faraday's law from an historical point of view. European Journal of Physics, 4, pp 170-179.

[5] PÉREZ, G.; MACEDO, B.; TORREGROSA, J.; SIFREDO, C.; VALDÉS, P. y VILCHES, A. 2005. ¿Cómo promover el interés por la cultura científica? Una propuesta didáctica fundamentada para la educación científica de jóvenes de 15 a 18 años. Chile. Andros Impresores. 